\title{
Huertos urbanos y periurbanos horizontales-verticales para el fomento de la educación ambiental sostenible
}

\author{
Juan G. Chipantiza-Masabanda, Amanda E. Bonilla-Bonilla, y Mario F. Jativa-Reyes \\ Escuela Superior Politécnica de Chimborazo, Sede Orellana, Facultad de Recursos Naturales, Puerto Francisco de \\ Orellana, Orellana 51002, Ecuador. (correo-e: juan.chipantiza@espoch.edu.ec; amanda.bonilla@espoch.edu.ec; \\ mario.jativa@espoch.edu.ec)
}

Recibido Ago. 3, 2020; Aceptado Oct. 6, 2020; Versión final Nov. 22, 2020, Publicado Abr. 2021

\begin{abstract}
Resumen
El objetivo del presente estudio es determinar la eficacia de un programa de formación basado en huertos urbanos y periurbanos horizontales-verticales en fomento de la educación ambiental sostenible de los estudiantes de agronomía de la Escuela Superior Politécnica de Chimborazo, sede Orellana-Ecuador. Se empleó el método deductivo, mediante un tipo de investigación explicativa fundamentada en un diseño cuasi experimental con grupo experimental y control, así como el uso de pre y pos prueba. Los resultados muestran significancia bilateral para el grupo experimental, lo que evidencia que el tratamiento aplicado fue efectivo en la muestra poblacional. Se concluye que los estudiantes se encuentran en capacidad de asumir la gerencia de proyectos de vinculación social con énfasis ambiental sustentable, en virtud de liderizar la organización de comunidades agrícolas urbanas, con la finalidad de promover la cultura endógena de crecimiento económico.
\end{abstract}

Palabras clave: aprendizaje activo; proyecto de educación; ecología; ecosistema; agricultura de subsistencia

\section{Vertical horizontal urban and peri-urban gardens to promote sustainable environmental education}

\begin{abstract}
The objective of this research study is to determine the effectiveness of a training program based on vertical horizontal urban and peri-urban gardens in promoting sustainable environmental education for agronomy students at the Polytechnic Superior School of Chimborazo, Orellana campus (Ecuador). The deductive method uses explanatory research based on a quasi-experimental design, with an experimental and control group, and pre- and post-tests. The results show that there was bilateral significance for the experimental group, suggesting that the applied treatment was effective in the population sample. It is concluded that students can manage social connection projects with emphasis on environmental sustainability by leading the organization of urban agricultural communities and by promoting endogenous economic growth culture.
\end{abstract}




\section{INTRODUCCIÓN}

La movilidad del mundo globalizado tecnológico, ante la masificación poblacional, busca alternativas de sostenibilidad del modelo económico en función de preservar la calidad de vida de las personas en consonancia con los estilos de vivencia social adquiridos en el presente siglo, así el enfoque sostenible para la vida promueve la educación ambiental - ecológica como eje transversal de la planificación pública y urbana, en función de promover conductas a favor de la preservación del medio ambiente desde una conciencia ecológica y planetaria (Aldana-Zavala y Colina-Ysea, 2019), haciendo indispensable la educación universitaria para el sostenimiento de los recursos naturales, con la finalidad de que estos sean distribuidos en equidad a la población, aunado al trabajo sistemático para la consecución de los objetivos de desarrollo sostenible (ODS), siendo su norte, erradicar la pobreza al 2030, lo ecológico - ambiental en conjunto con lo sostenible y sustentable, se conforman en paradigma para la transformación de la sociedad para crecer a través de prácticas no contaminantes del medio ambiente (Vallejos-Torres, et al., 2019).

La formación universitaria en todas sus carreras con énfasis en las relacionadas a las ciencias de la vida, deben procurar el fomento de una mentalidad de optimización y aprovechamiento de los recursos naturales en pro de su preservación, en razón de ser consecuente con prácticas profesionales cónsonas con una visión ciudadana planetaria (Rojas, et al., 2019), centrándose así en una perspectiva de trabajar en reciclaje para evitar la contaminación del medio ambiente como indicador de estilos ecológicos de convivencia social (Ramírez-Nieto, et al., 2019). En las facultades de agronomía debe promoverse el enfoque sostenible en funcionalidad transversal del aprendizaje (Andrade-Andrade, et al., 2019), generándose una pedagogía activa, a fin de formar a los estudiantes con base en la experiencia, promoviéndose competencias como la innovación, emprendimiento, con visión sostenible (Cabrera-Verdezoto, et al., 2017).

Se hace necesario el fomento de prácticas ambientales desde la formación universitaria, conociéndose el enfoque ambiental sostenible en razón de una praxis pedagógica experiencial - reflexiva, así como las estrategias posibilitadoras de concretar una sociedad sustentable fundamentada en lo ecológico (Pham, et al., 2019), siendo necesario involucrar a los estudiantes universitarios en la conformación de una perspectiva centrada en la preservación del medio ambiente (Espino-Román, et al., 2015). Tal formación debe ser concebida desde el paradigma de la complementariedad y la teoría del caos, fundamentando la interconexión de las acciones humanas y ambientales como la posibilidad de ser originadas en una determinada zona para afectar otras, esto a partir de lo planteado en el efecto mariposa (Pacheco, et al., 2020), siendo determinante formar para comprender la incertidumbre de un mundo complejo, es así que una práctica ecológica sostenible influirá positivamente en el medio ambiente local con efecto global.

Ante la crisis alimentaria y el deterioro ambiental tanto mundial como en el Ecuador, se promueve la siembra sostenible como una alternativa estratégica para la mitigación a estos problemas, en tal sentido, la práctica de las huertas urbanas y periurbanas se constituyen como una forma de optimizar los recursos naturales renovables y no renovables, enmarcado en un estilo de convivencia social, promoviéndose progresivamente un giro cultural en la sociedad ecuatoriana en virtud de consolidar una ciudadanía con visión sustentable de su entorno ecológico (Clavijo-Palacios y Cuvi, 2017), las universidades como extensión de las políticas de Estado, han iniciado un cambio paradigmático en el abordaje de las prácticas profesionales, pudiendo ser replicable esta experiencia a entornos rurales.

En tal sentido, la Escuela Superior Politécnica de Chimborazo (ESPOCH), sede Orellana-Ecuador, en la carrera de agronomía ha venido implementando la generación de huertos urbanos y periurbanos en razón de promover el enfoque sostenible de educación ambiental, por medio de las prácticas profesionales de los estudiantes. De este modo, se promueve no solo el conocimiento cognitivo, sino, habilidades, competencias, para la transformación del entorno ecológico de la universidad, teniendo la oportunidad de reconocer especies, enfermedades, métodos de propagación, a objeto de plantear alternativas nutricionales orgánicas que interactúan de forma natural en el espacio anti estrés, perciben el aroma natural de las flores y promueven la cosecha de productos frescos para una alimentación saludable, proyectándose un aprendizaje significativo en razón de enseñar la importancia del trabajo en equipo mediante la participación de los estudiantes en vinculación con la sociedad.

Los estudiantes de la Carrera de Agronomía que en la actualidad cursan sus estudios en la Escuela Superior Politécnica de Chimborazo Sede Orellana, no pueden formarse solo en conocimientos teóricos impartidos en las aulas, sino que se deben formarse mediante la implementación de métodos didácticos prácticos que generan interés en los estudiantes por lograr un aprendizaje significativo en función de transcender los retos del mundo global en función de las políticas alimenticias y de sostenibilidad al 2030. La iniciativa responde a problemas de los territorios urbanos como desnutrición, pobreza, desempleo, alimentos de mala calidad, entre otros. El producto generado en el huerto contribuye a que las familias aumentan la cantidad de proteína y calorías consumidas en comparación con las que no realizan tal actividad. Además, se mejoran los paisajes y la estética, por ende, la diversidad biológica se reconecta con la naturaleza y cuando se practica en escala, construye identidades urbanas más sustentables. 
A raíz de lo expuesto, se planteó el siguiente objetivo de investigación: Determinar la eficacia de un programa de formación basado en Huertos Urbanos y Periurbanos Horizontales y Verticales en fomento de la Educación Ambiental Sostenible de los estudiantes de agronomía de la Escuela Superior Politécnica de Chimborazo, sede Orellana-Ecuador.

\section{MÉTODO}

La sección metodológica se presenta en siete subsecciones: i) diseño de investigación; ii) ámbito espacial y geográfico de la investigación; iii) participantes; iv) procedimiento de la investigación; v) recolección de la información; vi) validez y confiabilidad del instrumento; y vii) técnica de análisis de información.

\section{Diseño de investigación}

Se empleó el método deductivo, mediante un tipo de investigación explicativa basado en diseño cuasi experimental con grupo experimental y control, con pre y pos prueba, con la intención de conocer las modificaciones cognitivas y conductuales de los participantes en relación al tratamiento aplicado. La población se conformó por 154 estudiantes de la carrera de agronomía de la Escuela Superior Politécnica de Chimborazo, sede Orellana-Ecuador.

\section{Ámbito espacial y geográfico de la investigación}

La Extensión Norte Amazónica de la ESPOCH contribuye activamente al desarrollo académico y social de la Provincia de Orellana y la Zona 2 (Napo, Orellana, Pichincha). La Región Amazónica es casi la mitad del territorio ecuatoriano, $45.1 \%$, (115.745 Km), pero solamente el $2 \%$ de la cuenca del Rio Amazonas involucra 8 países del subcontinente sudamericano y tienen una alta heterogeneidad territorial, caracterizados por la diversidad y complejidad de sus ecosistemas, los cuales son originados por sus características geomorfológicas, climatológicas y de formaciones vegetales.

\section{Participantes}

La muestra poblacional se dividió en 78 estudiantes para el grupo experimental (G1), mientras que 76 estudiantes conformaron el grupo control (G2). Ambos son estudiantes de la Extensión Norte Amazónica de la ESPOCH, en la carrera de agronomía, se destaca que (G1) son cursantes del décimo semestre, mientras que (G2), cursa noveno semestre, lo cual posibilita participar activamente en el tratamiento durante el próximo lapso académico. Tanto (G1) y (G2), fueron informados sobre su participación en el tratamiento, brindando su consentimiento para actuar en el mismo, datos que son empleados expresamente con fines investigativos.

\section{Procedimiento de la investigación}

Al grupo experimental (G1), se les aplicó el tratamiento basado en un programa de formación en Huertos Urbanos y Periurbanos Horizontales y Verticales en fomento de la Educación Ambiental Sostenible. Este programa, a su vez, estuvo constituido por los siguientes ejes de trabajo: 1. Educación ambiental sostenible, 2. Políticas ambientales del Ecuador, 3. Diseño de huertos urbanos y periurbanos, horizontales y verticales. 4. Identificación y desinfección de plagas 5. Producción sostenible y sustentable, 6 . Manejo sostenible del huerto, durante un lapso de 8 semanas continuas. Cada eje se encuentra conformado por temas que contribuyen a una formación integral del estudiante.

\section{Recolección de la información}

Semana 1: Al grupo experimental y control se aplicó pre y pos test. Semana 2 - 7: Aplicación del tratamiento. Semana 8: Prueba pre y pos test momento final. Se preparó una prueba tipo mixta para el pre y pos test, donde se evaluó el conocimiento, este instrumento estuvo conformado por 21 ítems de selección múltiple, correspondiente a los 6 ejes desarrollados en el tratamiento. Se complementó con una lista de cotejo en razón de comprobar las habilidades y competencias alcanzadas por los estudiantes, mediante demostración conductual y procedimental en trabajo de campo, sobre el desarrollo de huertos urbanos y periurbanos.

\section{Validez y confiabilidad del instrumento}

La prueba aplicada se validó a través del juicio de tres expertos, calculándose su confiabilidad mediante prueba piloto aplicada a 20 estudiantes con características similares a la población de estudio sin pertenecer a la misma, se calculó el coeficiente de Alfa de Cronbach obteniéndose un coeficiente de 0,93 catalogándose confiable para su aplicación. La lista de cotejo no fue validada por juicio de expertos ni fue calculada su coeficiente. 


\section{Técnica de análisis de información}

Así mismo, su contenido fue validado por el juicio de cinco expertos. Estadísticamente se procedió a aplicar la prueba T de Student para comparación de medias, conociéndose el valor de significancia para pre y pos test, mientras que para conocer cuál de los ejes de trabajo del programa de formación incidieron en mayor proporción, se aplicó prueba de ANOVA, con la finalidad de conocer cuál es el eje que resultó de mayor significancia para (G1), ambas pruebas se analizaron en apoyo del programa estadístico IBM SPSS versión 25.

\section{RESULTADOS}

Al existir significancia bilateral para el grupo experimental (G1POSTEST en Tabla 1) de 0,024 en comparación a los otros grupos que permanecieron en 0 , se evidencia cambio estadístico, lo cual implica que el tratamiento aplicado fue efectivo en la muestra poblacional. Tabla 1 presenta los resultados de la prueba T de Student de medias independientes para pre y pos test en muestra poblacional

Tabla 1: Prueba T de Student de medias independientes para pre y pos test en muestra poblacional

\begin{tabular}{|c|c|c|c|c|c|c|}
\hline & & & Prueba para un & muestra & & \\
\hline & & & Valor de pru & $b a=5$ & & \\
\hline & $T$ & $g l$ & Sig. (bilateral) & Diferencia de medias & $95 \%$ de inte & de confianza \\
\hline & & & & & Inferior & Superior \\
\hline G1PRETES & $-31,179$ & 77 & 0 & $-311,538$ & $-33,143$ & $-29,164$ \\
\hline G2PRETES & $-23,069$ & 75 & 0 & $-306,579$ & $-33,305$ & $-2,801$ \\
\hline G1POSTEST & $-2,297$ & 77 & 0,024 & $-0,0641$ & $-0,1197$ & $-0,0085$ \\
\hline G2POSTEST & $-63,952$ & 75 & 0 & $-376,316$ & $-38,804$ & $-36,459$ \\
\hline
\end{tabular}

\section{Prueba de hipótesis}

La prueba de hipótesis permitió cotejar el objetivo de la investigación, a partir del cambio estadístico producido en la prueba T de Student: H1: La aplicación de un programa de formación basado en Huertos Urbanos y Periurbanos Horizontales y Verticales en fomento de la Educación Ambiental Sostenible tuvo eficacia en los estudiantes de agronomía de la Escuela Superior Politécnica de Chimborazo, sede OrellanaEcuador. H0: La aplicación de un programa de formación basado en Huertos Urbanos y Periurbanos Horizontales y Verticales en fomento de la Educación Ambiental Sostenible no tuvo eficacia en los estudiantes de agronomía de la Escuela Superior Politécnica de Chimborazo, sede Orellana-Ecuador.

Se acepta $H 1$ y se rechaza $H O$. El nivel de significancia para la prueba ANOVA fue de 0,05 resultando el eje 3 "Diseño de huertos urbanos y periurbanos, horizontales y verticales (DHUP)" como el de mayor significancia sobre el grupo experimental en momento de pos test con un valor de 0,003

Tabla 2: Prueba ANOVA sobre ejes de trabajo y eficacia en muestra poblacional

\begin{tabular}{|c|c|c|c|c|c|c|}
\hline & & $\begin{array}{l}\text { Suma de } \\
\text { cuadrados }\end{array}$ & $g l$ & $\begin{array}{c}\text { Media } \\
\text { cuadrática }\end{array}$ & $F$ & Sig. \\
\hline \multirow[t]{3}{*}{ EAS } & Entre grupos & 0,009 & 1 & 0,009 & 0,024 & 0,876 \\
\hline & $\begin{array}{l}\text { Dentro de } \\
\text { grupos }\end{array}$ & 27,94 & 76 & 0,368 & & \\
\hline & Total & 27,949 & 77 & & & \\
\hline \multirow[t]{3}{*}{ PAE } & Entre grupos & 0,013 & 1 & 0,013 & 0,02 & 0,887 \\
\hline & $\begin{array}{l}\text { Dentro de } \\
\text { grupos }\end{array}$ & 47,282 & 76 & 0,622 & & \\
\hline & Total & 47,295 & 77 & & & \\
\hline \multirow[t]{3}{*}{ DHUP } & Entre grupos & 4,705 & 1 & 4,705 & 9,749 & 0,003 \\
\hline & $\begin{array}{l}\text { Dentro de } \\
\text { grupos }\end{array}$ & 36,679 & 76 & 0,483 & & \\
\hline & Total & 41,385 & 77 & & & \\
\hline
\end{tabular}


Tabla 2: continuación.

\begin{tabular}{|c|l|c|c|c|c|c|}
\hline & $\begin{array}{c}\text { Suma de } \\
\text { cuadrados }\end{array}$ & $g l$ & $\begin{array}{c}\text { Media } \\
\text { cuadrática }\end{array}$ & $F$ & Sig. \\
\hline IDP & Entre grupos & 0,001 & 1 & 0,001 & 0,001 & 0,974 \\
\hline & $\begin{array}{l}\text { Dentro de } \\
\text { grupos }\end{array}$ & 40,871 & 76 & 0,538 & & \\
\hline & Total & 40,872 & 77 & & & \\
\hline PSS & Entre grupos & 0,441 & 1 & 0,441 & 1,013 & 0,317 \\
\hline & $\begin{array}{l}\text { Dentro de } \\
\text { grupos }\end{array}$ & 33,047 & 76 & 0,435 & & \\
\hline MSH & Total & 33,487 & 77 & & & \\
\hline & Entre grupos & 0,068 & 1 & 0,068 & 0,164 & 0,686 \\
\hline & $\begin{array}{l}\text { Dentro de } \\
\text { grupos }\end{array}$ & 31,419 & 76 & 0,413 & & \\
\hline & Total & 31,487 & 77 & & & \\
\hline
\end{tabular}

\section{DISCUSIÓN}

La discusión se plantea en razón de los ejes de trabajo contentivos del tratamiento aplicado, los cuales fueron mencionados en el apartado método. El eje de educación ambiental sostenible, desarrollado en el tratamiento aplicado, contribuye a la formación de un estudiante en capacidad de visibilizar la perspectiva de un mundo en equilibrio natural, por cuanto el ser humano asume practicas fundamentadas en promover la sostenibilidad del medio ambiente (Taylor, et al., 2018), lo cual se corresponde con lo planeado por (Andrade-Andrade, et al., 2019), al indicar la necesidad formar para un enfoque sostenible de convivencia ciudadana. Tal aseveración conduce a la conformación de una sociedad basada en valores ambientales como fundamento de su accionar en razón de proyectar un sistema educativo en donde la generación de conocimientos se hace en base al trabajo cooperativo desde una visión sistémica, pensada en las consecuencias generadas desde una mala actuación humana en detrimento del ambiente (De-Leijster, et al., 2019), relacionándose con lo descrito por (Cabrera-Verdezoto, et al., 2017), sobre una pedagogía activa con fundamentación en lo ambiental para concienciar al estudiante sobre una actuación profesional apegada a lo ecológico.

Otro tema abordado en el tratamiento, se refirió a las políticas ambientales del Ecuador, promoviéndose el trabajo cooperativo y sostenible como una fuente de encuentro entre el ser humano y el medio ambiente, proyectándose el buen vivir como paradigma de convivencia para propiciar una agricultura productiva en función de preservar la calidad de vida (Ma, et al., 2018), focalizándose además con el planteamiento de (Aldana-Zavala y Colina-Ysea, 2019), al trabajarse en la conformación de una visión global ciudadana - ecológica, articulándose una cultura agrícola en favor de generar aportes positivos a fin de minimizar el impacto global sobre el cambio climático (Marshall, et al., 2015), considerándose para tal fin, la generación de estrategias complementarias que contribuyen desde el Estado Ecuatoriano a la agenda climática 2030, siendo así que las universidades ha asumido la formación desde una visión sostenible concerniente a las carreras relacionadas con las ciencias de la tierra, concatenándose con la perspectiva de (Clavijo-Palacios y Cuvi, 2017), esta acción posibilita además una educación ambiental fundamentada en la sinergia de la epistemología de saberes ancestrales y conocimientos científicos con la finalidad de promover el Sumak Kawsay como política esencial del Estado Ecuatoriano (ColladoRuano, et al., 2020).

El diseño de huertos urbanos y periurbanos tanto horizontales como verticales, favorecen de modo integral, la formación de los estudiantes, quienes, mediante sus prácticas profesionales, tienen la oportunidad de trabajar en función de construir el conocimiento desde la sinergia cognitiva y procedimental, promoviendo así sus competencias en pro de descubrir sus habilidades mediante el aprendizaje activo y la fusión de la teoría y la práctica (François, et al, 2019). Así se promueve una educación innovadora en donde el emprendimiento se conjuga en activar el liderazgo de un estudiante capaz de proyectar una economía basada en lo sostenible como alternativa de crecimiento social, de este modo, al elevarse la producción de alimentos en entornos urbanos, se hace posible articular comunidades en capacidad de ser auto sostenibles (Kamel-Louhichi, et al., 2018).

La identificación y desinfección de plagas, es un eje transversal en la carrera de agronomía, por ende, los estudiantes al establecer los huertos urbanos como práctica profesional, afinan sus competencias en la detección y erradicación de plagas desde un contexto sostenible, proyectándose además la transferencia de 
conocimientos a la comunidad para articular proyectos de vinculación social (Bellwood-Howard, et al., 2020). La idea es trabajar en la tecnificación alternativa, es decir, con instrumentos y medios no invasivos o contaminantes que proyecten la unión del conocimiento científico con el empírico de la sabiduría ancestral, situación compleja en favor de promover el paradigma de la complementariedad en el aprendizaje agronómico (Glover, et al., 2019), siendo esta temática favorable para germinar una cultura de abordaje del ecosistema agrícola en razón de una visión sostenible de la sociedad, articulándose con lo planteado por (Pacheco, et al., 2020).

La producción sostenible y sustentable conduce a la conformación de un estudiante con capacidad para diseñar los escenarios agrícolas en función de ser productivos, rentables, con el menor impacto a la naturaleza, y proyectando el consumo de alimentos de calidad en beneficio de la salud, siendo concomitante con una perspectiva cooperativa de economía social (Sumberg, et al., 2020). La dimensión sostenible, conlleva a repensar el accionar agrícola en función de cumplir con la demanda alimenticia en tiempos de COVID-19, existiendo un reto no solo de producir, sino, de transportar los alimentos, sobre todo cuando existen países cuyo producto interno bruto, básicamente depende de los ingresos obtenidos por la venta de rubros agrícolas, por lo tanto, lo sostenible involucra el abordaje de estrategias para optimizar el proceso de producción y consumo de alimentos en concordancia con los retos 2030 (Baudron, et al., 2020).

El manejo sostenible del huerto, constituye la sinergia de los ejes descritos anteriormente, generándose las competencias gerenciales y administrativas con fines de constituir un liderazgo promotor de una economía sustentable (Laage-Thomsen, et al., 2020). La educación universitaria en este sentido, acciona mecanismos curriculares con la finalidad de promover estrategias pedagógicas en consecución de un aprendizaje basado en competencias, de ese modo, se precisa la formación de un estudiante proactivo y emprendedor, posibilitado para transcender el modelo económico basado exclusivamente en el salario, por uno donde se incentive el empleo desde la auto gestión empresarial (Calvet-Mir, et al., 2019).

La educación ambiental sostenible también coadyuva a la conformación de comunidades organizadas para auto gestionar los recursos naturales con los cuales cuenta, potenciando el agrupamiento en organizaciones locales con vinculación al desarrollo de las políticas públicas en función de realizar proyectos productivos bajo una visión sostenible. En consecuencia, se fomenta el liderazgo local en función de alcanzar la conformación de nuevos espacios habitables desde donde se toman decisiones que permiten establecer el buen vivir como paradigma social a partir de la visión cooperativa de trabajo mancomunado (Franchini, et al., 2017).

La complejidad del abordaje ambiental como eje transversal sustentada en los objetivos de desarrollo sostenible 2030 en conformidad de erradicar la pobreza, requiere la sinergia de múltiples factores entre los cuales resaltan el conocimiento, la organización social y el aprendizaje rizómico que converge como factor esencial, por cuanto permite al estudiante confluir en un pensamiento sistémico en interconexión con la realidad ambiental (Unander, et al., 2020). En tal razón, desde la universidad, se articula con la comunidad en procura de su organización siendo posible esto, por medio de la formación de los agentes sociales en virtud de constituir líderes en las localidades donde se trabaje de la mano para su crecimiento integral, proyectándose la interconexión de los diversos eventos que conforman un fenómeno, así el estudiante toma conciencia de la importancia de su actuación, por cuanto sus consecuencias inciden sobre otros, tal como se hace mención en la teoría del caos.

El liderazgo social con carácter ambiental sostenible es posible afrontarlo en la medida que se concatena lo científico con el saber popular, representado por estudiantes, docentes y agricultores nativos. Así puede promoverse el diseño, ejecución, de proyectos sostenibles en función de establecer una cultura sustentable desde lo agrícola. Vale destacar que la educación en este sentido, toma una pertinencia que permite confrontar el currículo tradicional con uno en donde se aborda desde el constructivismo social, es decir, la generación no solo de conocimientos, sino de procedimientos y técnicas en la mejora continua de la sociedad (Jeon, et al., 2019).

Por consiguiente, el sistema educativo es fundamental; por ello, la educación a partir de lo abordado, debe pasar por una reingeniería curricular, con la intención de promover la perspectiva de ciudadanía planetaria, siendo esto un factor esencial para lograr, en los próximos años, la consolidación de una gestión ambiental fundamentada en salvar la vida en el planeta. Para tal fin, es necesario además promover una percepción bioética del sujeto como agente protagonista en compañía de los seres vivos, por tal razón, el enfoque de individualidad debe minimizarse por uno en donde lo colectivo, la cooperación, el trabajo en equipo y la sinergia puedan ser parte del accionar social en razón de lograr, desde la práctica, la competencia intercultural como elemento esencial de la vida humana (Tate, 2018).

Desconocer lo ambiental, lo ecológico, la educación, la política, la economía, la cultura, la agricultura y la salud como factores que se interrelacionan entre sí, es condenar al aislamiento disciplinar que se ha 
mantenido como un factor relegado en la sociedad, situación que permite transcender al enfoque de la complejidad como acción de entretejer las diversas aristas planteadas en un eje medular para lograr el diseño de políticas ambientales que puedan dar respuestas concretas a los diversos problemas sociales y ambientales que no solo vive Latinoamérica, sino, el mundo (Pau, et al., 2020).

Tales retos deben ser planteados en las diversas legislaciones con la finalidad de atacar las problemáticas en contribución de accionar desde la educación ciudadana, sinergia que permite la construcción de una perspectiva para trabajar progresivamente en la consecución de una mejor sociedad, por lo cual, lo ambiental devela problemáticas sociales que en el entretejido socio político abren la posibilidad de generar proyectos en donde la participación pública y privada se complementen para favorecer el devenir de los próximos años y así presentar metas concretas de cara al 2030, la cual se proyecta como fecha límite para lograr el abordaje del cambio climático y políticas ambientales en favor de una nueva sociedad basada en lo ecológico para su preservación (Matsushita, et al., 2018). La economía desde sus diversos ámbitos, debe girar en torno a promover una sociedad basada en la equidad y el equilibrio ecológico que producen sus acciones sobre el medio ambiente (Konstantinidis, 2018).

\section{CONCLUSIONES}

En prosecución de los resultados, así como su constatación teórica con investigaciones relacionadas a las variables de investigación, se conciben las sucesivas conclusiones primordiales: 1. La eficacia del programa al ser significativa en la muestra experimental, permitió establecer cambios cognitivos y conductuales en los estudiantes con la finalidad de abordar en su ejercicio profesional, el enfoque de educación ambiental sostenible en razón de contribuir a la conformación de una sociedad productiva y sustentable. 2 . Los estudiantes tienen la posibilidad a partir de lo abordado en el tratamiento, actuar en base las premisas del enfoque sostenible - sustentable para la preservación del medio ambiente, esto posibilita la construcción donde el imaginario social se conduce a actuar para la consolidación de una cultura basada en lo ecológico, de ese modo, se contribuye desde la universidad a la conformación de una persona integral desde la concepción cognitiva, social, espiritual, ecológica, para actuar profesional por un mundo de convivencia por el respeto mutuo entre las personas con la naturaleza, lo que abre el debate sobre la interculturalidad ambiental.

\section{AGRADECIMIENTO}

Este trabajo se realizó gracias al apoyo, colaboración y paciencia de muchas personas. Se agradece a la Escuela Superior Politécnica de Chimborazo Sede Orellana, que incentivó la realización de la investigación en cooperación con docentes y estudiantes de la Carrera de Agronomía y, en especial, a los estudiantes que participaron dentro del Proyecto de Vinculación "Huertos".

\section{REFERENCIAS}

Aldana-Zavala, J., y Colina-Ysea, F. Marketing verde en la conformación de una ciudadanía planetaria en el ámbito educativo latinoamericano. http://dx.doi.org/10.36097/rsan.v0i31.972, Revista San Gregorio, 3,150-161. (2019).

Andrade-Andrade, V., Gorozabel-Muñoz, W., Mendoza-Rivadeneira, F., y Arteaga-Solórzano, R. Conservación de sábila (Aloe vera) y mango (Mangífera indica L) mediante almíbar de miel de abeja, Agroecología Global. Revista Electrónica de Ciencias del Agro y Mar, 1(1), 66-83. (2019).

Baudron, F., y Liégeois, F. Fixing our global agricultural system to prevent the next COVID-19, https://doi.org/10.1177/0030727020931122, Outlook on Agriculture, 49, 111-118. (2020).

Bellwood-Howard, I., y Ripoll, S. Divergent understandings of agroecology in the era of the African Green Revolution, https://doi.org/10.1177/0030727020930353, Outlook on Agriculture, 49(2), 103-110. (2020).

Cabrera-Verdezoto, Rodrigo P, Vera-Oyarzún, M, Echeverría-Pezoa, Rodrigo A, Amtmann-Moyano, C, y Pinochet-Tejos, D. Caracterización de los agricultores urbanos en la comuna de Valdivia, Región de los Ríos, Chile. https://dx.doi.org/10.4067/S0718-34292017005000012, Idesia (Arica), 35, 27-32. (2017).

Calvet-Mir, L., y March, H. Crisis and post-crisis urban gardening initiatives from a Southern European perspective: The case of Barcelona, https://doi.org/10.1177/0969776417736098, European Urban and Regional Studies, 26, 97-112. (2019).

Clavijo-Palacios, C, y Cuvi, N. La sustentabilidad de las huertas urbanas y periurbanas con base agroecológica: el caso de Quito, https://dx.doi.org/10.17141/letrasverdes.21.2017.2608, Letras Verdes, Revista Latinoamericana de Estudios Socioambientales, 21, 68-91. (2017).

Collado-Ruano, J., Falconí-Benitez, F., y Malo-Larrea, A. Educación ambiental y praxis intercultural desde la filosofía ancestral del Sumak Kawsay, Utopía y Praxis Latinoamericana, 25(90), 120-135. (2020).

De-Leijster, V, João-Santos, M, y otros seis autores. Agroecological management improves ecosystem services in almond orchards within one year, https://doi.org/10.1016/j.ecoser.2019.100948, Ecosystem Services, Volume 38, 100948. (2019). 
Espino-Román, P, Olaguez-Torres, E, y Davizon-Castillo, Y. Análisis de la Percepción del Medio Ambiente de los Estudiantes de Ingeniería en Mecatrónica, https://dx.doi.org/10.4067/S0718-50062015000400006, Formación universitaria, 8, 45-54. (2015).

Franchini, M., Viola E., y Barros-Platiau Ana. The challenges of the anthropocene: from international environmental politics to global governance, https://doi.org/10.1590/1809-4422asoc214v2022017, Ambiente \& Sociedade, 20, 177-202. (2017).

François, J, Dessart, J, y Barreiro-Hurlé, R. Behavioural factors affecting the adoption of sustainable farming practices: a policy-oriented review, https://doi.org/10.1093/erae/jbz019, European Review of Agricultural Economics, 46, 417471. (2019).

Glover, D., Sumberg, J., Ton, G., Andersson, J., y Badstue, L. Rethinking technological change in smallholder agriculture, https://doi.org/10.1177/0030727019864978, Outlook on Agriculture, 48, 169-180. (2019).

Jeon, J. Invisibilizing politics: Accepting and legitimating ignorance in environmental sciences, https://doi.org/10.1177/0306312719872823, Social Studies of Science, 49, 839-862. (2019).

Kamel-Louhichi, P, Maria-Espinosa, A, Gomez-y-Paloma, S. Economic impacts of CAP greening: application of an EUwide individual farm model for CAP analysis (IFM-CAP), https://doi.org/10.1093/erae/jbx029, European Review of Agricultural Economics, 45, 205-238. (2018).

Konstantinidis, C. Capitalism in Green Disguise: The Political Economy of Organic Farming in the European Union, https://doi.org/10.1177/0486613417717482, Review of Radical Political Economics, 50, 830-852. (2018).

Laage-Thomsen, J., y Blok, A. Varieties of green: On aesthetic contestations over urban sustainability pathways in a Copenhagen community garden, https://doi.org/10.1177/2514848620902806, Environment and Planning E: Nature and Space. (2020).

Ma, W., Abdulai, A. y Goetz, R. Agricultural Cooperatives and Investment in Organic Soil Amendments and Chemical Fertilizer in China, https://doi.org/10.1093/ajae/aax079, American J. of Agricultural Economics, 100: 502-520. (2018).

Marshall, E., Aillery, M., Malcolm, S. y Williams, R. Agricultural Production under Climate Change: The Potential Impacts of Shifting Regional Water Balances in the United States, https://doi.org/10.1093/ajae/aau122, Amer. J of Ag. Econ., 97: 568-588. (2015).

Matsushita, K., Taki, H., Yamane, F. y Asano, K. Shadow Value of Ecosystem Resilience in Complex Natural Land as a Wild Pollinator Habitat, https://doi.org/10.1093/ajae/aax075, American J. of Agricultural Economics, 100: 829-843 (2018).

Pacheco, P. Estudio de un proceso de aprendizaje en ciencias experimentales a partir de la Teoría del Caos, https://dx.doi.org/10.4067/S0718-50062020000300077, Formación universitaria, 13, 77-88. (2020).

Paul, B. K., Groot, J. C., Maass, B. L., Notenbaert, A. M., Herrero, M., \& Tittonell, P. A. Improved feeding and forages at a crossroads: Farming systems approaches for sustainable livestock development in East Africa, https://doi.org/10.1177/0030727020906170, Outlook on Agriculture, 49, 13-20. (2020).

Pham, Trung T., Lobos, G., y Vidal-Silva, C. Innovación en Minería de Datos para el Tratamiento de Imágenes: Agrupamiento K-media para Conjuntos de Datos de Forma Alargada y su Aplicación en la Agroindustria, https://dx.doi.org/10.4067/S0718-07642019000200135, Información tecnológica, 30, 135-142. (2019).

Ramírez-Nieto, N, Baena, Y, y Osorio, C. Caracterización Fisicoquímica de Emulsiones Aceite/Agua a partir de Uchuva (Physalis peruviana) como Ingrediente para la Industria Alimenticia, https://dx.doi.org/10.4067/S071807642019000300147, Información tecnológica, 30, 147-156. (2019).

Rojas, A, Rodríguez-Barona, S, y Montoya, J. Evaluación de Alternativas de Aprovechamiento Energético y Bioactivo de la Cáscara de Plátano, https://dx.doi.org/10.4067/S0718-07642019000500011, Información tecnológica, 30, 1124. (2019).

Sumberg, J., y Sabates-Wheeler, R. Children's work in African agriculture: Time for a rethink, https://doi.org/10.1177/0030727020930330, Outlook on Agriculture, 49, 99-102. (2020).

Tate, D. A. A High Achieving Inner-City School: An Ecological Analysis, https://doi.org/10.1177/0042085918794778, Urban Education. (2018).

Taylor, P, Grass, I, Alberts, A, Joubert, E, Tscharntke, T. Economic value of bat predation services - A review and new estimates from macadamia orchards, https://doi.org/10.1016/j.ecoser.2017.11.015, Ecosystem Services, 30, 372-381. (2018).

Unander, T. E., y Sørensen, K. H. Rhizomic learning: How environmental non-governmental organizations (ENGOs) acquire and assemble knowledge, https://doi.org/10.1177/0306312720908343, Social Studies of Science. (2020).

Vallejos-Torres, G, Arévalo, L, lliquin, I, y Solis, R. Respuesta en Campo de Clones de Café a la Inoculación con Consorcios de Hongos Micorrízicos Arbusculares en la Región Amazonas, Perú, https://dx.doi.org/10.4067/S071807642019000600073, Información tecnológica, 30, 73-84. (2019). 